Fabio Reale et al.

Science 341, 251 (2013);

DOI: $10.1126 /$ science.1235692

This copy is for your personal, non-commercial use only.

If you wish to distribute this article to others, you can order high-quality copies for your colleagues, clients, or customers by clicking here.

Permission to republish or repurpose articles or portions of articles can be obtained by following the guidelines here.

The following resources related to this article are available online at www.sciencemag.org (this information is current as of July 19, 2013 ):

Updated information and services, including high-resolution figures, can be found in the online version of this article at:

http://www.sciencemag.org/content/341/6143/251.full.html

This article cites $\mathbf{3 6}$ articles, 1 of which can be accessed free:

http://www.sciencemag.org/content/341/6143/251.full.html\#ref-list-1

This article appears in the following subject collections:

Astronomy

http://www.sciencemag.org/cgi/collection/astronomy 


\section{Bright Hot Impacts by Erupted Fragments Falling Back on the Sun: A Template for Stellar Accretion}

\author{
Fabio Reale, ${ }^{1,2 *}$ Salvatore Orlando, ${ }^{2}$ Paola Testa, ${ }^{3}$ Giovanni Peres, ${ }^{1,2}$ \\ Enrico Landi, ${ }^{4}$ Carolus ]. Schrijver ${ }^{5}$
}

Impacts of falling fragments observed after the eruption of a filament in a solar flare on 7 June 2011 are similar to those inferred for accretion flows on young stellar objects. As imaged in the ultraviolet (UV)-extreme UV range by the Atmospheric Imaging Assembly onboard the Solar Dynamics Observatory, many impacts of dark, dense matter display uncommonly intense, compact brightenings. High-resolution hydrodynamic simulations show that such bright spots, with plasma temperatures increasing from $\sim 10^{4}$ to $\sim 10^{6}$ kelvin, occur when high-density plasma $\left(>>10^{10}\right.$ particles per cubic centimeter) hits the solar surface at several hundred kilometers per second, producing high-energy emission as in stellar accretion. The high-energy emission comes from the original fragment material and is heavily absorbed by optically thick plasma, possibly explaining the lower mass accretion rates inferred from x-rays relative to UV-optical-near infrared observations of young stars.

$\mathrm{M}$ ass accretion from the circumstellar disk onto the stellar surface plays an important role in the late phases of star formation $(1,2)$. Young low-mass stars are presumably connected to and interact with circumstellar disks through magnetic funnels (3). The plasma accretes along the funnels at nearly freefall velocity (hundreds of kilometers/second) onto the central young star. Most evidence for accretion originates from the region of impact of the disk material onto the stellar chromosphere and includes infrared and optical excess emission in lines and continuum (4-6) and a soft (energy < $0.7 \mathrm{keV}$ ) x-ray excess above the coronal emission from dense $\left(10^{11}\right.$ to $\left.10^{13} \mathrm{~cm}^{-3}\right)$ and hot (2 to $\left.4 \mathrm{MK}\right)$ plasma (7-9). Hot impact spots are observed in photospheric emission (10).

Current models suggest that the impact region is rather complex because of the interplay between radiation and hydrodynamics $(11-14)$ : The streams might be highly structured in both density and velocity, leading to inhomogeneous impact spots; the impacting material is heated to millions of degrees by kinetic energy dissipation and might partially sink into the chromosphere, its emission being substantially absorbed by the thick chromosphere and by the dense falling material itself. The impact can also drive strong motions and feed material into surrounding coronal

${ }^{1}$ Dipartimento di Fisica e Chimica, Università di Palermo, Piazza del Parlamento 1, 90134 Palermo, Italy. ${ }^{2}$ Istituto Nazionale di Astrofisica (INAF)/Osservatorio Astronomico di Palermo, Piazza del Parlamento 1, 90134 Palermo, Italy. ${ }^{3}$ Harvard-Smithsonian Center for Astrophysics, 60 Garden Street, MS 58, Cambridge, MA 02138, USA. ${ }^{4}$ Department of Atmospheric, Oceanic and Space Sciences, University of Michigan, Ann Arbor, MI 48109, USA. ${ }^{5}$ Lockheed Martin Advanced Technology Center, Palo Alto, CA 94304, USA.

*Corresponding author. E-mail: reale@astropa.unipa.it structures (14), as recently suggested for the classical T Tauri star TW Hydrae (accretion-fed corona) $(15,16)$. The density and velocity of the accreting material determine the temperature of the hot plasma and the sinking depth (13). This complexity hampers a deep understanding of the dynamical and radiative properties of the plasma in the impact region and, ultimately, of the accretion processes in young stars.

Here, we study plasma impacts observed after the solar flare on 7 June 2011 at 6 UT that show interesting analogies with inferred stellar accretion impacts. The flare was categorized as " $\mathrm{M}$ class" on the Global Online Enrollment System scale of the National Oceanic and Atmospheric Administration. It was observed by the ultraviolet (UV) and extreme UV (XUV) narrowband channels of the Atmospheric Imaging Assembly (AIA) (17) on board the Solar Dynamics Observatory (SDO) (18), with high spatial resolution ( $\sim 0.6$ arc sec per pixel) and high cadence (12 s) (19) (see supplementary materials, section S1). During the flare, we clearly see, in all SDO/AIA XUV channels, that a dense, dark filament is broken and violently ejected (movie S1). The cloud propagates outward at a speed of several hundred kilometers per second and fragments in all directions. The fastest fragments escape in the form of a typical coronal mass ejection (20), observed by the Solar and Heliospheric Observatory/Large Angle and Spectrometric Coronagraph (21) white light telescope. Slower fragments fall back onto the solar surface. They are visible in all AIA XUV channels as dark, irregular, and moving strips on the brighter background corona. During their fall, the fragments change their morphology, stretching and dividing further (19), but they generally maintain a coherent structure and remain dark throughout their trajectory. When the fragments hit the solar surface, they drive intense brightenings in the impact region, visible in all AIA XUV and UV channels. Here, we focus our attention on these impacts (movies S2 and S3).

Before hitting the surface, many downfalling fragments follow similar parabolic trajectories (movie S2); one trajectory was traced to be close to a free fall from combining AIA and STEREOSECCHI EUVI (22) images. We have measured several impact speeds to be in the range of 300 to $450 \mathrm{~km} / \mathrm{s}$ (see supplementary materials, section S1.2), similar to typical stellar accretion flow speeds (23). We know that the fragments are dense and cool, because we see them in absorption in the XUV channels. From the amount of absorption, we constrain their density in the range of 2 to $10 \times 10^{10} \mathrm{~cm}^{-3}$ with a temperature of $\sim 3 \times 10^{4} \mathrm{~K}$ (see supplementary materials, section S1.1). The impacts are dispersed over a large fraction of the solar surface (Fig. 1). The typical cross section of impacting fragments is around 2000 to $4000 \mathrm{~km}$, whereas the long side of elongated fragments can extend to $>10^{4} \mathrm{~km}$. The duration of the brightenings ranges between 1 and $\geq 6 \mathrm{~min}$ in the XUV and UV spectra. During each brightening, the XUV emission typically increases by factors of 2 to 5 over the unperturbed conditions.

According to magnetograms measured with the SDO/Helioseismic and Magnetic Imager (HMI) (fig. S4), the trajectories of the falling fragments and most impacts occur in regions where the magnetic field is rather weak. Most fragments do not decelerate while falling, and the ram and thermal pressures of the fragments at the impacts are estimated to be substantially larger than the local magnetic pressure (supplementary materials, section S1.3). Thus, we can assume that the magnetic field does not play an important role in the impacts, and we can describe the plasma evolution with a purely hydrodynamic model of plasma blobs, falling in a low-density corona with an impact speed of $400 \mathrm{~km} / \mathrm{s}$ and a density of $5 \times 10^{10} \mathrm{~cm}^{-3}$ (both in the measured ranges). We ran several two-dimensional simulations (cylindrical geometry) to match the observed variety of cases: a spherical droplet (with a radius of $2000 \mathrm{~km}$ ), a train of droplets, and an elongated fragment (stream). An accurate description of the evolution required a very high spatial resolution (down to $\sim 5 \mathrm{~km}$; see supplementary materials, section S2.1).

In our simulations, the very dense model fragments fell freely in the tenuous ambient atmosphere with a faint bow shock preceding them. As they hit the chromosphere, they penetrated and smashed against the layers whose pressure was equal to the ram pressure of the infalling fragment (movie S4). The single spherical droplet was soon squashed to a thin, dense, and hot layer and then bounced back, forming an outward surge that expanded at a speed of $\sim 200 \mathrm{~km} / \mathrm{s}$. The surge reached temperatures above $10^{5} \mathrm{~K}$, but it cooled down in a few seconds, due to the rapid expansion and radiative losses. 
For comparison with observations, we computed the emission that would be detected with the AIA XUV channels where we expected the plasma to be mostly optically thin; that is, 94, 131, 171, 193, 211, and $335 \AA$. In agreement with the observation, the emission evolution was similar in all the channels (Fig. 1 and movie S5). The plasma became visible in this channel when it rose above the transition region while it bounced back. Although the surge expanded over several tens of seconds, the plasma remained bright for a much shorter time ( $20 \mathrm{~s})$ (Fig. 1) and then faded out rapidly, due to the cooling and rarefaction. The brightening region also remained small, not much larger than the initial size of the droplet; it was the internal part of the outward surge and was almost featureless and hemispherical. The plasma did not rise substantially above the surface. We traced that most of the emission came from the original impacting droplet, with very little contribution from the ambient material.

The simulations with the train of droplets and the stream showed longer-living (to more than $100 \mathrm{~s}$ ) and larger-scale (up to $\sim 10,000 \mathrm{~km}$ ) bouncing structures (movies S6 to S9). Although the emitting plasma was in a shell, integration along the line of sight kept the emission concentrated along the central axis. The longer-lasting stream emission was structured in sequences of evolving blobs and fringes (Fig. 1).

The morphology of the emission in the simulations matches the observations, up to the later phases when coupling to the surrounding magnetic field becomes important. The size of the emitting region and the temporal evolution of the emission in the simulations are in quantitative agreement with the observations. The observed light curves (fig. S5) have shapes, flux, and time scales similar to those derived from the simulations (fig. S9). The observed brightenings generally last longer than the simulated ones, as can be expected because the impacting fragments may be much more extended than the modeled ones or can be made by showers of smaller-scale blobs, slightly displaced from one another.

Simulations either with a much smaller density of the falling droplet $\left(5 \times 10^{9} \mathrm{~cm}^{-3}\right)$ (movie $\mathrm{S} 10)$ or a much smaller impact speed $(250 \mathrm{~km} / \mathrm{s})$
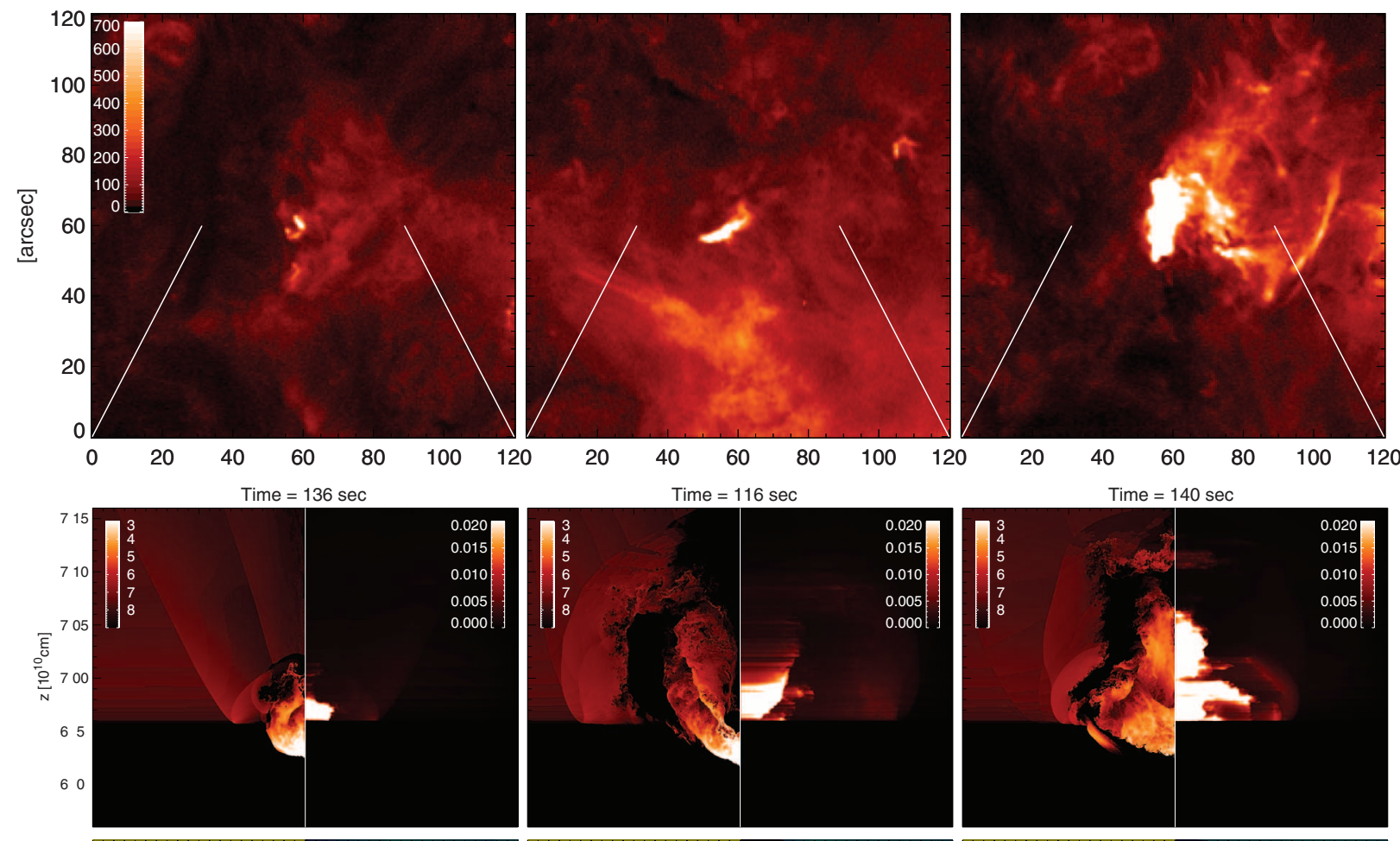

Time $=140 \mathrm{sec}$
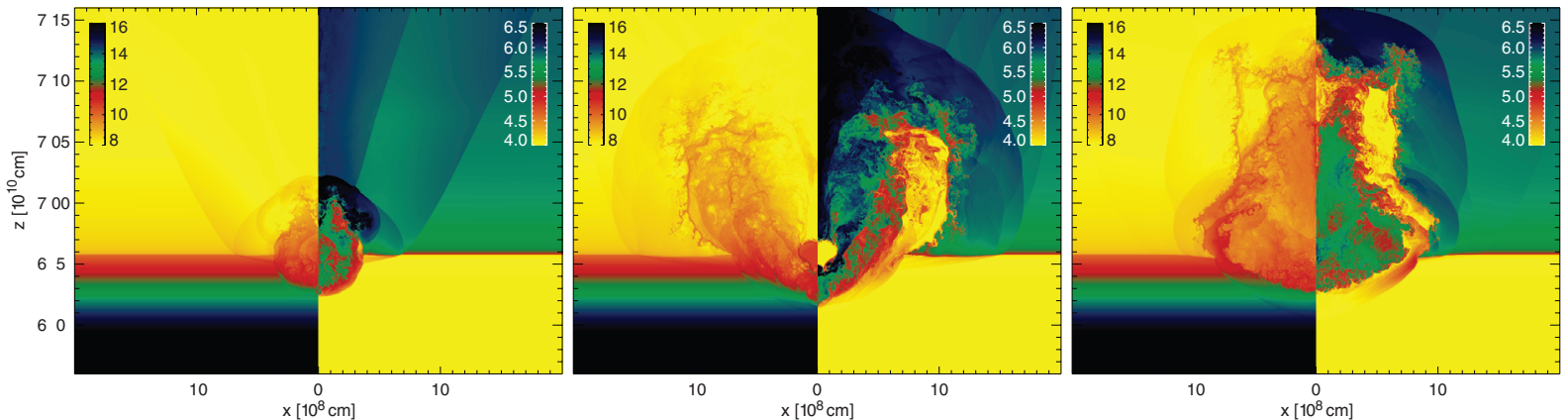

Fig. 1. Observed and modeled impact brightenings. (Top) Observed impacts of dense fragments erupted after an M-class solar flare on 7 June 2011 in the SDO/AIA 171 A channel. Three cases are shown, representative of smallto large-scale impact brightenings (from left to right, taken at 7:26:25, 7:29:02, and 08:09:49 UT, with the plot origin at $[x, y]=\left[357^{\prime \prime},-132^{\prime \prime}\right],\left[468^{\prime \prime}\right.$, $\left.-137^{\prime \prime}\right]$, [335", $\left.-130^{\prime \prime}\right]$ from the disk center). The color palette is data number (DN) per second per pixel. The white lines indicate how the width of the lower rows scales to the first row. (Middle) Simulated impacts of fragments that may drive the respective brightenings (from left to right: droplet, train of droplets,

and stream) with a hydrodynamic model (at the labeled times since the beginning of the simulation). The left side of each map is a cross section of the emission in the $171 \AA$ channel (logarithmic scale, $\mathrm{DN} \mathrm{cm} \mathrm{cm}^{-3} \mathrm{~s}^{-1}$ ), and the right side is the emission integrated along the horizontal line of sight; i.e., tangential to the solar surface (4223 DN per pixel per second). When integrating, we exclude the heavily absorbed emission; i.e., by plasma at high density $\left(>10^{10} \mathrm{~cm}^{-3}\right)$ or below the transition region (see supplementary materials, section S2.2). (Bottom) Cross sections of the logarithm of the density (left, per cubic centimeter) and temperature (right, kelvin). 
(movie S11) were unable to produce substantial emission (fig. S9A), indicating that the scenario is coherent. Thus, our hydrodynamic simulations show that the hot and bright impacts after the eruption are caused by the high density $\left(\gg>10^{10} \mathrm{~cm}^{-3}\right)$ and speed (free-fall speed, 300 to $450 \mathrm{~km} / \mathrm{s}$ ) of the downfalling debris. These values are close to those of the plasma involved in stellar accretion flows.

For most stellar accretion flows [see (15) for an exception], the mass accretion rates derived from x-rays are consistently lower (by one or more orders of magnitude) than the corresponding rates derived from UV-optical-near infrared observations (24-26). According to our analysis of a solar event, the impact of the dense fragments leads to detectable high-energy emission. From the model, we find that the mass of plasma responsible for the brightenings in the $171 \AA$ channel (see supplementary materials, section S3) ranges between 5 and $30 \%$ of the original mass of the fragments. This is mainly because of the absorption by the optically thick chromosphere and/or by the dense part of the material outflowing after the impact (depending on the orientation of the impact region with respect to the line of sight). In addition, the simulations tell us that droplets with low density or low velocity are unable to produce substantial emission; the observations tell us that some of the falling fragments produce no detectable brightening. Therefore, both the absorption of x-ray emission from dense plasma and the wide range of velocity and density values of the infalling fragments contribute to underestimating the mass accretion rate from the XUV band.

Our simulations also show that the XUV emission arises from the original impacting material. Although our fragmented solar downflows differ from the conceptual stellar accretion flows (which are hypothesized to be continuous streams, chan- neled by the magnetic field), the structure of the impact region (presenting hot plasma partially rooted in the chromosphere) is similar in the two cases. Moreover, as suggested recently (19), the accretion flows are likely to be frayed, even when they are magnetically confined and stream along straight tubes. Therefore, the dynamics and energetics spatially resolved in the solar observations are a template and laboratory to study accretion processes in astrophysics.

\section{References and Notes}

1. D. Lynden-Bell, J. E. Pringle, Mon. Not. R. Astron. Soc. 168, 603-637 (1974).

2. L. Hartmann, Accretion Processes in Star Formation, Cambridge Astrophysics Series (Cambridge Univ. Press, New York, 1998).

3. A. Koenigl, Astrophys. J. 370, L39-L43 (1991).

4. C. Bertout, G. Basri, J. Bouvier, Astrophys. J. 330, 350-373 (1988)

5. A. Natta, L. Testi, S. Randich, Astron. Astrophys. 452 , 245-252 (2006)

6. G. J. Herczeg, L. A. Hillenbrand, Astrophys. J. 681 , 594-625 (2008).

7. J. H. Kastner, D. P. Huenemoerder, N. S. Schulz, C. R. Canizares, D. A. Weintraub, Astrophys. J. 567, 434-440 (2002).

8. A. Telleschi, M. Güdel, K. R. Briggs, M. Audard, L. Scelsi, Astron. Astrophys. 468, 443-462 (2007).

9. C. Argiroffi, A. Maggio, G. Peres, Astron. Astrophys. 465, L5-L8 (2007).

10. J.-F. Donati et al., Mon. Not. R. Astron. Soc. 386, 1234-1251 (2008).

11. ]. J. Drake, 13th Cambridge Workshop on Cool Stars, Stellar Systems and the Sun, vol. 560 of ESA Special Publication, F. Favata, G. A. J. Hussain, B. Battrick, Eds. (European Space Agency, Noordwijk, 2005), p. 519.

12. H. M. Günther, J. H. M. M. Schmitt, ]. Robrade, C. Liefke, Astron. Astrophys. 466, 1111-1121 (2007).

13. G. G. Sacco et al., Astron. Astrophys. 522, A55 (2010).

14. S. Orlando et al., Astron. Astrophys. 510, A71 (2010).

15. N. S. Brickhouse, S. R. Cranmer, A. K. Dupree, G. J. M. Luna, S. Wolk, Astrophys. J. 710, 1835-1847 (2010).

16. A. K. Dupree et al., Astrophys. J. 750, 73 (2012).

17. J. R. Lemen et al., Sol. Phys. 275, 17-40 (2012).

18. W. D. Pesnell, B. J. Thompson, P. C. Chamberlin, Sol. Phys. 275, 3-15 (2012).
19. D. E. Innes, R. H. Cameron, L. Fletcher, B. Inhester, S. K. Solanki, Astron. Astrophys. 540, L10 (2012).

20. X. Cheng et al., Astrophys. J. 745, L5 (2012).

21. G. E. Brueckner et al., Sol. Phys. 162, 357-402 (1995)

22. ].-P. Wuelser et al., Society of Photo-Optical Instrumentation Engineers (SPIE) Conference Series, S. Fineschi, M. A. Gummin, Eds. (SPIE, San Diego, 2004), vol. 5171, pp. 111-122.

23. N. Calvet, E. Gullbring, Astrophys. J. 509, 802-818 (1998)

24. E. Gullbring, L. Hartmann, C. Briceno, N. Calvet, Astrophys. J. 492, 323-341 (1998).

25. J. Muzerolle, N. Calvet, C. Briceño, L. Hartmann, L. Hillenbrand, Astrophys. J. 535, L47-L50 (2000).

26. R. L. Curran et al., Astron. Astrophys. 526, A104 (2011)

Acknowledgments: We thank B. De Pontieu for help and suggestions. F.R., G.P., and S.O. acknowledge support from Italian Ministero dell'Università e Ricerca and Agenzia Spaziale Italiana, contract I/023/09/0. P.T. was supported by contract SP02H1701R from Lockheed-Martin to the Smithsonian Astrophysical Observatory. P.T. and C.J.S. are supported by NASA contract NNG04EA00C for the SDO AIA. E.L. is supported by NASA grants NNX11AC20G and NNX10AQ58G and NSF grant AGS-1154443. S.O. acknowledges partial support from the INAF. The software used in this work was, in part, developed by the U.S. Department of Energy-supported Advanced Simulation and Computing/Alliance Center for Astrophysical Thermonuclear Flashes at the University of Chicago. We acknowledge the CINECA Award HP10CEG9MW and INAF/Osservatorio Astronomico di Palermo for high-performance computing resources and support. SDO data were supplied courtesy of the SDO/HMI and SDO/AIA consortia. SDO is the first mission to be launched for NASA's Living With a Star Program. See the supplementary materials for details on the data, data analysis, and modeling.

Supplementary Materials

www.sciencemag.org/cgi/content/full/science.1235692/DC1

Materials and Methods

Supplementary Text

Figs. $\mathrm{S1}$ to $\mathrm{S9}$

Table S1

References (27-40)

Movies S1 to S18

28 January 2013; accepted 5 June 2013

Published online 20 June 2013;

$10.1126 /$ science. 1235692

\section{Switchable Static and Dynamic Self-Assembly of Magnetic Droplets on Superhydrophobic Surfaces}

\author{
Jaakko V. I. Timonen, ${ }^{1 *} \dagger$ Mika Latikka, ${ }^{1}$ Ludwik Leibler, ${ }^{2}$ Robin H. A. Ras, ${ }^{1 *}$ Olli Ikkala ${ }^{1 *}$
}

Self-assembly is a process in which interacting bodies are autonomously driven into ordered structures. Static structures such as crystals often form through simple energy minimization, whereas dynamic ones require continuous energy input to grow and sustain. Dynamic systems are ubiquitous in nature and biology but have proven challenging to understand and engineer. Here, we bridge the gap from static to dynamic self-assembly by introducing a model system based on ferrofluid droplets on superhydrophobic surfaces. The droplets self-assemble under a static external magnetic field into simple patterns that can be switched to complicated dynamic dissipative structures by applying a time-varying magnetic field. The transition between the static and dynamic patterns involves kinetic trapping and shows complexity that can be directly visualized.

$\mathrm{F}$ unctional patterns and structures are essential in a wide variety of natural and engineered systems (1). They often form of small subunits by autonomous self-assembly, which is driven by free-energy gradients (2). Static selfassembly denotes a process in which the system reaches an energy minimum (equilibrium) wherein the ordered structure appears. Archetypical examples are structured block copolymers $(3,4)$, nanoparticles $(5,6)$, nanorods (7), liquid crystals $(8)$, and hierarchical supramolecular systems (9). They find applications in data storage (10) and structural colors (11), for instance. On the other hand, dynamic self-assembly denotes a process in which the structure forms when the system is forcefully kept away from an energy minimum (out of equilibrium) by continuous energy supply and dissipation $(12,13)$. Dynamic self-assembly is most notably encountered in biological systems (14-16),

${ }^{1}$ Department of Applied Physics, Aalto University (formerly Helsinki University of Technology), P.O. Box 15100, FI-02150 Espoo, Finland. ${ }^{2}$ Matière Molle et Chimie, UMR 7167 CNRSESPCI, Ecole Supérieure de Physique et Chimie Industrielles, 10 rue Vauquelin, 75005 Paris, France.

*Corresponding author. E-mail: jaakko.timonen@aalto.fi (J.V.I.T.); robin.ras@aalto.fi (R.H.A.R.); olli.ikkala@aalto.fi (O.I.) †Present address: Non-Equilibrium Energy Research Center, Northwestern University, 2145 Sheridan Road, Evanston, IL 60208, USA. 